Y.-H. Chu, N.B. Suntzeff, J.E. Hesser, and D.A. Bohlender, eds.

\title{
Kinematics of $F$ to $M$ Supergiants in the 30 Dor and Shapley II Regions of the Large Magellanic Cloud
}

\author{
E. Maurice, N. Martin \\ Observatoire de Marseille 2, Place Le Verrier F-13248 Marseille Cedex \\ 04, France \\ G. Testor, M.C. Lortet \\ Observatoire de Meudon, DAEC, F-92195 Meudon Cedex, France
}

\begin{abstract}
.
Accurate radial velocities have been obtained with the CORAVEL photoelectric scanner at ESO, La Silla, for 36 F-M supergiants in the 30 Dor and Shapley II regions of the LMC $\left(5^{\mathrm{h}} 20^{\mathrm{m}}<\mathrm{RA}(2000)<5^{\mathrm{h}} 50^{\mathrm{m}}\right.$, $\left.-70^{\circ} 09^{\prime}<\operatorname{Dec}(2000)<-68^{\circ} 30^{\prime}\right)$. On the basis of these data, we revisit the content and spatial extent of the four kinematical groups of F-M supergiants discovered in this area by Prévot et al. 1989. Each is the oldest part of a larger star forming region, recognized by young HII regions or $\mathrm{CO}$ clouds, and/or a surrounding $\mathrm{H} \alpha+[\mathrm{NII}]$ superbubble. We discuss various gas tracers including the interstellar sodium and calcium lines.
\end{abstract}

In order to study the structure in depth and age of this interesting region of the LMC, we used different sets of data: accurate radial velocities of FM supergiants, the HI survey at Parkes telescope by Rohlfs et al. (1984) and Luks et al. (1992), and additional HI data of better sensivity at 9 positions by Meaburn et al. (1987). These data along with the high resolution NaI and CaII ISM observations in front of $35 \mathrm{OB}$ stars allowed us to correct the description of the HI gas distribution in the quoted survey (especially towards the S-E of the field and the HI poor center of Shapley II). In addition, OB stars with known velocity and data on CO clouds (Cohen et al. 1988; Johansson et al. 1998) and the associated dust allowed a first hint on the depth structure in the region under study. We obtained revised parameters of the four kinematical groups of F-M supergiants and we attempted to relate each of them to a larger star formation region.

- Shapley II (276.3 \pm 3.5 (s.e.) $\left.\mathrm{km} \mathrm{s}^{-1}, \mathrm{~N}=41\right)$ inside the superbubble LMC3 (Meaburn 1980) and 30 Dor $1\left(288.7 \pm 3.9 \mathrm{~km} \mathrm{~s}^{-1}, \mathrm{~N}=11\right)$ are related to one or two distinct extended HI features of similar velocities in which the star formation nearly exhausted the gas.

- 30 Dor $2\left(266.4 \pm 4.4 \mathrm{~km} \mathrm{~s}^{-1}, \mathrm{~N}=26\right)$ extends from $-69^{\circ} 30^{\prime}$ to $\mathrm{LH} 90$ and the core of the 30 Dor nebula and probably to the North and East to Sk-68 ${ }^{\circ} 135$, $\mathrm{Sk}-69^{\circ} 270, \mathrm{Sk}-69^{\circ} 247$. L1 designates this complex. It is obscured in all its S-E part by a large amount of dust linked to CO clouds. 


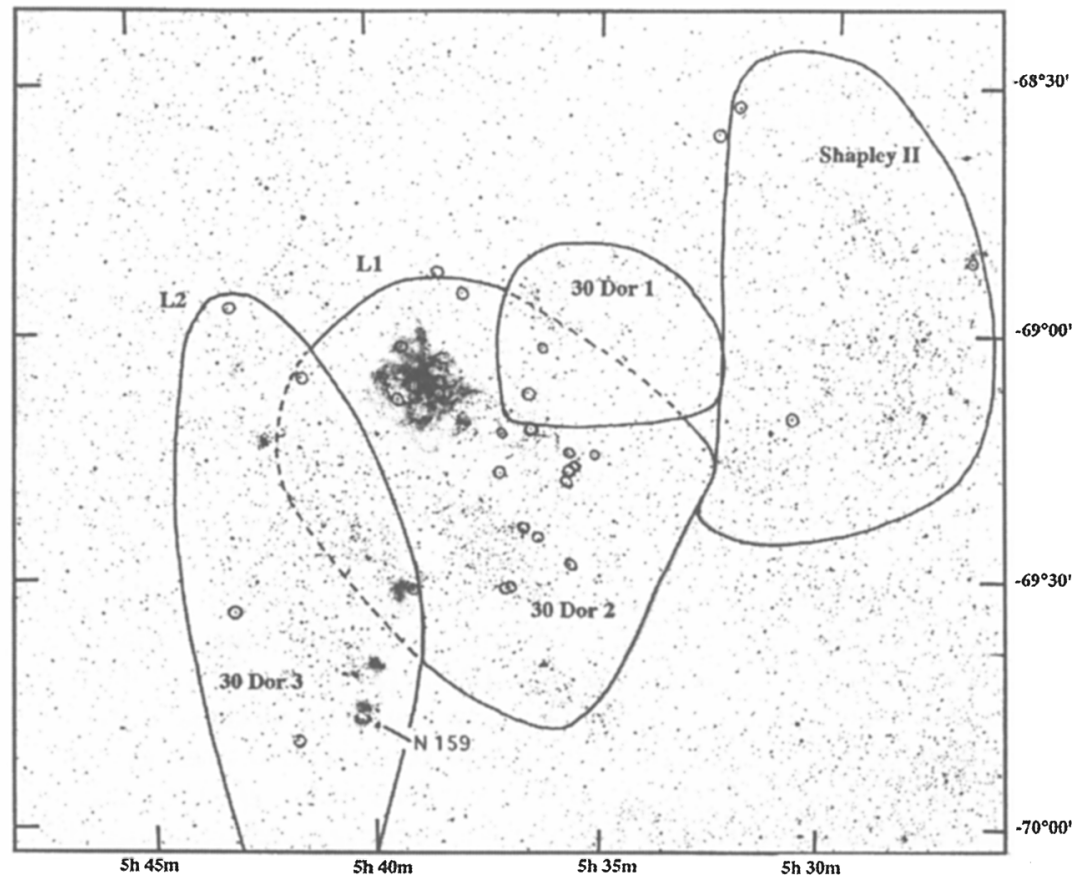

Figure 1. Schematic view of the structure of the 30 Dor and Shapley II regions. The four kinematical groups of F-M supergiants, Shapley II, 30 Dor 1, 30 Dor 2 and 30 Dor 3 are indicated inside the (very approximate) boundaries of the associated star forming regions. Open circles indicate the OB stars we used.

- 30 Dor $3\left(249.7 \pm 5.1 \mathrm{~km} \mathrm{~s}^{-1}, \mathrm{~N}=14\right)$ is the oldest part of a star forming region orientated North-South, here labelled L2, rich in young HII regions and CO clouds around N159 (Johansson et al. 1998).

We show that L1 lies behind L2 and behind the HI cloud related to 30 Dor 1. More details on this work will be given in a forthcoming paper.

\section{References}

Cohen, R.S., et al. 1988, ApJ, 331, L95

Johansson, L.E.B., et al. 1998, A\&A, 331, 857

Luks, T., \& Rohlfs, K., 1992, A\&A, 263, 41

Meaburn, J. 1980, MNRAS, 192, 365

Meaburn, J., Marston, A.P., McGee, R.X., \& Newton, L.M. 1987, MNRAS, 225, 591.

Prévot, L., Rousseau, J., \& Martin, N. 1989, A\&A, 225, 303

Rohlfs, K., Kreitschmann, J., Siegmar, B.C., \& Feitzinger, J.V. 1984, A\&A, 137, 343. 